\title{
Dimensional accuracy of camera casing models 3D printed on Mcor IRIS: A case study
}

\author{
Mandić, M. ${ }^{a}$, Galeta, T. ${ }^{\text {a, }}{ }^{,}$, Raos, P. ${ }^{a}$, Jugović, V. $^{\mathrm{a}}$ \\ ${ }^{a}$ Mechanical Engineering Faculty in Slavonski Brod, J.J. Strossmayer University of Osijek, Slavonski Brod, Croatia
}

\begin{abstract}
A B S T R A C T
The main objective of this research was to determine the deviations and evaluate the dimensional accuracy of 3D printed camera casing models compared to the original models in the STL format. The study sample consisted of the 3D printed camera casing models and the same models in the STL format. The STL format came from Mcor in a set of sample models shipped with the 3D printer. The models were 3D printed on Mcor IRIS and then scanned with ATOS 3D scanner. A comparison between the scanned and original STL models was made in the GOM Inspect software. The results indicate that the maximum deviation occurred on the scanned front camera cover and it is $0.82 \mathrm{~mm}$ in the direction $\mathrm{z}$. The average deviation of scanned front camera cover is $0.0845 \mathrm{~mm}$ and the average deviation of scanned back camera cover is 0.0722 $\mathrm{mm}$. The analysis of the results proves that the three-dimensional printed paper-based parts have the dimensions close to the original CAD models.
\end{abstract}

(c) 2016 PEI, University of Maribor. All rights reserved.

\section{ARTICLE INFO}

Keywords:

Additive manufacturing

3D printing

Mcor IRIS

3D scanning

Accuracy

*Corresponding author:

tgaleta@sfsb.hr

(Galeta, T.)

Article history:

Received 14 April 2016

Revised 6 October 2016

Accepted 10 October 2016

\section{References}

[1] Farzadi, A., Waran, V., Solati-Hashjin, M., Rahman, Z.A.A., Asadi, M., Osman, N.A.A. (2015). Effect of layer printing delay on mechanical properties and dimensional accuracy of 3D printed porous prototypes in bone tissue engineering, Ceramics International, Vol. 41, No. 7, 8320-8330, doi: 10.1016/j.ceramint.2015.03.004.

[2] Farzadi, A., Solati-Hashjin, M., Asadi-Eydivand, M., Abu Osman, N.A., (2014). Effect of layer thickness and printing orientation on mechanical properties and dimensional accuracy of 3D printed porous samples for bone tissue engineering, PLOS ONE, Vol. 9, No. 9, e108252, doi: 10.1371/journal.pone.0108252.

[3] Galantucci, L.M., Bodi, I., Kacani, J., Lavecchia, F. (2015). Analysis of dimensional performance for a 3D opensource printer based on fused deposition modeling technique, Procedia CIRP, Vol. 28, 82-87, doi: 10.1016/ j.procir.2015.04.014.

[4] Galeta, T., Kljajin, M., Karakasic, M. (2008). Geometric Accuracy by 2-D Printing Model, Strojniški Vestnik - Journal of Mechanical Engineering, Vol. 54, No. 10, 725-733.

[5] Daneshmand, S., Aghanajafi, C., Shahverdi, H. (2013). Investigation of rapid manufacturing technology effect on aerodynamics properties (Istraživanje utjecaja tehnologije brze izrade na aerodinamička svojstva), Tehnički vjesnik - Technical gazette, Vol. 20, No. 3, 425-433.

[6] Scuzs, T.D., Brabazon, D. (2007). Analysis of the Effects of 3DP Parameters on Part Feature Dimensional Accuracy, In: Proceedings of the Solid Freeform Fabrication Symposium, Austin, Texas, USA, 20-31.

[7] Asadi-Eydivand, M., Solati-Hashjin, M., Farzad, A., M., Osman, N.A.A. (2016). Effect of technical parameters on porous structure and strength of 3D printed calcium sulfate prototypes, Robotics and Computer-Integrated Manufacturing, Vol. 37, 57-67, doi: 10.1016/i.rcim.2015.06.005.

[8] Islam, M.N., Boswell, B., Pramanik, A. (2013). An investigation of dimensional accuracy of parts produced by three-dimensional printing, In: Proceedings of the World Congress on Engineering, London, United Kingdom, 522525.

[9] Fahad, M., Hopkinson, N. (2012). A new benchmarking part for evaluating the accuracy and repeatability of additive manufacturing (AM) processes, In: $2^{\text {nd }}$ International Conference on Mechanical, Production and Automobile Engineering, Singapore, 234-238. 
[10] Mcor Technologies, Mcor IRIS HD Features \& Specs, from http://mcortechnologies.com/3d-printers/iris/ accessed August 1, 2015.

[11] Tomasiak, J. (2012). Rapid technology in industrial fitting, In: Annals of DAAAM for 2012 \& Proceedings of the $23^{\text {rd }}$ International DAAAM Symposium, Vol. 23, No. 1, 433-436.

[12] GOM, ATOS Compact Scan, from http://www.gom.com/metrology-systems/system-overview/atos-compactscan.html accessed August 4, 2015.

[13] Meshmixer., MeshmixerManual, from http://meshmixer.com/help/manual/MeshmixerManual.pdf, accessed August 4, 2015.

[14] GOM, GOM Inspect Software, from http://www.gom.com/3d-software/gom-inspect.html, accessed August 4, 2015.

[15] Krolczyk, G.M., Legutko, S. (2014). Experimental analysis by measurement of surface roughness variations in turning process of duplex stainless steel, Metrology and Measurement Systems, Vol. 21, No. 4, doi: 10.2478/mms$\underline{2014-0060 .}$. 


\section{APEM}

\title{
Dimenzijska natančnost modelov ohišja za kamero 3D, tiskanega na Mcor IRIS: Študija primera
}

\author{
Mandić, M. ${ }^{a}$, Galeta, T. ${ }^{\text {a, }}$, Raos, P. ${ }^{a}$, Jugović, V. ${ }^{\text {a }}$ \\ ${ }^{a}$ Mechanical Engineering Faculty in Slavonski Brod, J.J. Strossmayer University of Osijek, Slavonski Brod, Croatia
}

\section{POVZETEK}

Glavani cilj raziskave je določiti odstopanja in ovrednotiti dimenzijsko natančnost 3D tiskanih modelov ohišja za kamero v primerjavi z originalnimi modeli v STL formatu. Vzorce za raziskavo predstavljajo 3D tiskana ohišja za kamero in njihovi modeli v STL formatu. STL format kamere je eden izmed primerov Mcor, ki so bili poleg 3D tiskalnika. Modeli so bili natisnjeni na Mcor IRIS tiskalniku in skenirani z ATOS 3D skenerjem. Primerjava med skeniranimi in originalnimi STL modeli je bila izvedena v GOM Inspect programskem okolju. Rezultati kažejo, da se največja dimenzijska odstopanja pojavijo na sprednjem pokrovu kamere in znašajo 0,82 $\mathrm{mm} v$ smeri $z$. Povprečno dimenzijsko odstopanje sprednjega pokrova kamere znaša $0,0845 \mathrm{~mm}$, povprečno dimenzijsko odstopanje zadnjega pokrova kamere pa $0,0722 \mathrm{~mm}$. Analiza rezultatov dokazuje, da imajo trodimenzionalno natisnjeni izdelki temelječi na papirju dimenzije blizu originalnim iz CAD modelov.

(C) 2016 PEI, University of Maribor. All rights reserved.

\section{PODATKI O ČLANKU}

Ključne besede:

Dodajalne tehnologije

3D tiskanje

Mcor IRIS

3D skeniranje

Natančnost

* Kontaktna oseba: tgaleta@sfsb.hr (Galeta, T.)

Zgodovina članka: Prejet 14. aprila 2016

Popravljen 6. oktobra 2016

Sprejet 10. oktobra 2016 